\title{
Relación entre el Aprendizaje Autorregulado y Rendimiento Académico en Estudiantes de Ingeniería Industrial de Universidades de Trujillo 2017
}

\author{
Miguel Enrique Alcalá Adrianzén, Magister Investigación y Docencia Universitaria ${ }^{1}$, Alexander Manuel Villoslada Chilón, \\ Magister Investigación y Docencia Universitaria ${ }^{2},{ }^{1}$ Universidad Privada del Norte, Perú, miguel.alcala@upn.edu.pe \\ ${ }^{2}$ Universidad Privada del Norte, Perú, alexander.villoslada@upn.pe
}

\begin{abstract}
Resumen- Los conceptos de aprendizaje autorregulado son relevantes en un proceso que comprende las relaciones de trabajo, formación profesional y sociedad. Durante la pandemia del COVID19 con la virtualidad de la enseñanza fue más atractivo su estudio. El objetivo de la investigación fue establecer la relación que existe entre el aprendizaje autorregulado y el rendimiento académico en estudiantes de Ingeniería Industrial del V, VI, VII y VIII ciclo en el semestre académico 2017-I, en universidades de Trujillo, aplicándose el Inventario de Aprendizaje Autorregulado (SRLI) Norabuena 2011, una versión adaptada de Lindner, Harris y Gordon 1992. Para el análisis se utilizó el coeficiente de correlación de Pearson, a un nivel de significancia de 0.01 . Los resultados obtenidos en la matriz de correlaciones, en la universidad V es 0.777 $y$ en la universidad $N$ es 0.854 y en conjunto es 0.822. Estos resultados permitieron concluir que existe una relación positiva y estadísticamente significativa para el nivel de aprendizaje autorregulado en su escala total, así como las subescalas: ejecutiva, cognitiva, motivación y control de ambiente, y el nivel de rendimiento académico, según la prueba de correlación de Pearson. Los resultados de la investigación implican un apoyo a los docentes para trasformar su rol en el aula.
\end{abstract}

Palabras clave: Aprendizaje autorregulado, metacognición, motivación, rendimiento académico.

Abstract- The concepts of self-regulated learning are relevant in a process involving the relationships of work, professional training and society. During the COVID-19 pandemic with the virtuality of teaching, its study became more attractive. The objective of the research was to establish the relationship that exists between self-regulated learning and academic performance in students of Industrial Engineering of the V,VI, VII and VIII cycle in the academic semester 2017-I, in universities of Trujillo, applying the Self-Regulated Learning Inventory (SRLI) Norabuena 2011, an adapted version of Lindner, Harris and Gordon 1992. Pearson's correlation coefficient was used for the analysis, at a significance level of 0.01. The results obtained in the correlation matrix, in university $V$ is 0.777 and in university $N$ is 0.854 and overall is 0.822 . These results allowed concluding that there is a positive and statistically significant relationship for the level of self-regulated learning in its total scale, as well as the subscales: executive, cognitive, motivation and environmental control, and the level of academic performance, according to Pearson's correlation test. The results of the research imply a support to teachers to transform their role in the classroom.

Keywords-- Self-regulated learning, metacognition, motivation, academic performance

Digital Object Identifier (DOI):

http://dx.doi.org/10.18687/LEIRD2021.1.1.21

ISSN: 2414-6390 ISBN: 978-958-52071-9-6

\section{INTRODUCCION}

El estudio del aprendizaje autorregulado es relevante en el escenario de pandemia Covid 19 [1], muy útil en un proceso complejo que comprende las relaciones de trabajo, formación profesional y la comunidad [2]. Durante la pandemia del Covid 19 , se evidenciaron factores preocupantes para los docentes como el bajo rendimiento académico, problemas financieros, dudas vocacionales y problemas emocionales. Las consecuencias fueron una búsqueda de apoyo directo a los docentes que desean trasformar su rol en el aula.

El rendimiento académico es el producto del aprendizaje; la forma en que el aprendizaje se define de manera operativa, debido al constructo psicológico que no es observable y medible de forma directa [3]. La nota de acceso a la universidad es considerada como la variable de mayor incidencia en los resultados académicos, al menos en los primeros años de universidad [4]. La complejidad del Rendimiento Académico que no es un producto centrado en el estudiante o el docente o en su interacción, sino que es el resultado de múltiples variables de tipo personal y social, motiva a los profesionales en educación, ciencias sociales y psicología educativa a analizar, describir y evaluar [5].

El aprendizaje autorregulado, se trata de un constructo que comprende varios campos de investigación (por ejemplo, motivación, cognición y metacognición), con sus propias definiciones y problemas propios [6]. Es la autorregulación como el control que el estudiante realiza en sus pensamientos, acciones, emociones y motivaciones, mediante estrategias personales para lograr los objetivos que se ha propuesto [7]. El modelo socio cognitivo de Zimmerman es considerado como el más completo y a la vez el más específico [8].

La referencia [9] realizaron un estudio en carreras de licenciatura e ingeniería, de la Universidad Virtual del Tecnológico de Monterrey, México, determinaron que los estudiantes tuvieron buen rendimiento académico, independientemente de su estilo de aprendizaje, identificando un alto grado de aprendizaje auto regulado. Observaron que la diferencia radica en el locus de control, así los estudiantes de bajo rendimiento académico indicaron causas ajenas, en cambio el estudiante exitoso identificó causas que encuentra en él mismo.

La referencia [10] docentes de la Universidad de Navarra, España, determinaron qué si bien el docente y estudiante comparten la responsabilidad del aprendizaje, está también el 
énfasis en el rol del docente como diseñador y facilitador del aprendizaje de sus estudiantes. Identificaron también la necesidad del aprendizaje autorregulado y de manera autónoma ante la sociedad del conocimiento. Esta situación determinó que el modelo de enseñanza tuvo implicancias para las funciones del docente, y que se necesitó suficiente capacitación para ejercerlo.

La referencia [11] en el estudio para los estudiantes de educación superior, Puebla, México; el rendimiento académico, se ubicó en los extremos de alto y bajo, y que tuvo diferencias en la forma que se llevó el proceso de autorregulación del aprendizaje. El análisis de la información la realizaron en función de las 16 categorías de análisis según la teoría [12].

La referencia [13] en la Universidad Rafael Landívar de Guatemala, concluyeron qué por estar los coeficientes de correlación, lejos de la unidad (0.372), no existe relación entre las técnicas de autorregulación y el rendimiento académico de los adolescentes. El investigador aplicó el Cuestionario de Autorregulación para el Aprendizaje Académico, adaptado [14], el cual está conformado por 20 preguntas para evaluar: conciencia metacognitiva activa, control y verificación, esfuerzo diario en tareas y procesamiento activo en clases.

La referencia [15] en el estudio realizado en la Universidad Nacional Santiago Antúnez de Mayolo - Huaraz, para la correlación del aprendizaje autorregulado y rendimiento académico, el coeficiente de Correlación de Pearson determinado fue 0,6769 para estudiantes de enfermería y 0,7055 en obstetricia; a un nivel de significancia de $\mathrm{p}<0,05$ concluyó que existe relación significativa positiva. En la recopilación de datos utilizó el Inventario de Aprendizaje Autorregulado (SRLI) [16] en la versión 4.01. El cuestionario con 60 ítems y puntaje máximo de 300 puntos.

La referencia [17] en estudiantes de Ingeniería Industrial de la Universidad Tecnológica del Perú, determinó una la relación significativa $(\mathrm{p}<0.01)$, para el coeficiente de correlación de Pearson de 0,596, entre el aprendizaje autorregulado y el rendimiento académico. Para la recolección de datos utilizó el Inventario de Aprendizaje Autorregulado (SRLI) [16], con 80 ítems y un puntaje máximo de 400 puntos

La referencia [18] en los estudiantes de psicología de tres universidades de Arequipa, según el modelo de Kolb, determinaron que el estilo divergente fue el predominante y altos porcentajes de estudiantes tuvieron niveles bajos de metacognición, autoconocimiento, autorregulación y evaluación.

La referencia [19] en el uso de las estrategias metacognitivas y el aprendizaje autorregulado en estudiantes del Instituto Superior Pedagógico Indoamérica, en Trujillo, determinó un coeficiente de correlación alto, de $\mathrm{r}=0,767$; el coeficiente de correlación entre la autoestima y el aprendizaje autorregulado fue de $r=0,934$ altamente significativo, puesto que el p-valor asociado fue de p =0,000. En el estudio utilizó tres instrumentos validados: $\mathrm{El}$ inventario de estrategias metacognitivas [20], el Inventario de Aprendizaje
Autorregulado (SRLI) [14] y el inventario de autoestima forma C, [21].

La referencia [22] determinó en los estudiantes de Educación Secundaria de las Instituciones Educativas del distrito de Taurija, La Libertad, el coeficiente de correlación de Pearson $\mathrm{r}=0,529$, para la correlación entre las variables estrategias didácticas y rendimiento académico, una correlación positiva media; el coeficiente de Pearson fue $r=0,688$, para la correlación entre las variables estrategias de aprendizaje y rendimiento académico, una correlación positiva media $\mathrm{La}$ técnica que utilizó fue la encuesta, y el instrumento empleado un cuestionario de tipo Likert. Para el rendimiento académico utilizó las escalas del Ministerio de Educación del Perú establecido en el Diseño Curricular Nacional [23].

La referencia [24], sostienen qué los sistemas de aseguramiento de calidad en las universidades monitorearon los indicadores de insumo mostrando los esfuerzos para mejorar la calidad del servicio (tales como la capacitación docente, la infraestructura y fondos para la investigación); mientras que los indicadores de resultado fueron el reflejo de dichos esfuerzos, como la inserción laboral o la producción científica. Se evidenció así, una profunda preocupación en el proceso enseñanza aprendizaje de la educación superior.

En la ciudad de Trujillo, las universidades, en la última década han visto la necesidad de hacer que el estudiante, se sienta el personaje principal y constructor de su propio aprendizaje. Reconocer la importancia de reformular su modelo pedagógico, plan curricular y capacitación docente en las teorías psicopedagógicas contemporáneas del aprendizaje.

En la ciudad de Trujillo cinco universidades tienen la escuela profesional de ingeniería industrial [25], en el reporte estadístico para el año 2016, los estudiantes matriculados en esta especialidad son: Universidad Nacional De Trujillo - UNT, 797; Universidad Privada Cesar Vallejo - UCV, 11013; Universidad Privada del Norte - UPN, 11321; Universidad Católica de Trujillo Benedicto XVI - UCT, 193 y Universidad Privada Antenor Orrego - UPAO, 2382. Pero solo en las tres primeras la escuela de Ingeniería Industrial tiene más de 10 años de funcionamiento. Dos de ellas tienen el mayor número de estudiantes, además al 2021 UPN, UNT y UPAO están acreditadas por ICACIT y solo UPN cuenta con la acreditación ABET. Con la pandemia COVID-19 se evidenció la necesidad de innovar en el proceso de aprendizaje autorregulado.

El fomento del aprendizaje autorregulado es una meta vital para el estudiante de ingeniería industrial dónde debe aprender a aprender y a razonar de manera autónoma, crítica, estratégica y meta cognitivamente. En la educación superior del Perú faltan investigaciones de este tipo, y es posible que se asuma que en este nivel los estudiantes poseen mejores estrategias de aprendizaje asimiladas.

En ese contexto el presente estudio apunta, a determinar la relación entre el aprendizaje autorregulado y el rendimiento académico de estudiantes de Ingeniería Industrial en las universidades de Trujillo. Para el aprendizaje autorregulado se considera las subescalas: ejecutiva, cognitiva, motivación y 
control del ambiente. La importancia del estudio radica a que existen implicancias en la labor del docente, quien deberá formarse adecuadamente para atender estos cambios Así también, adquirir conocimiento sobre el proceso de facilitación e incrementar sus habilidades como facilitadores, por medio de estrategias de aprendizaje que el docente y el estudiante deben implementar.

\section{METODOLOGÍA}

\section{A. Población y muestra}

En la ciudad de Trujillo se tiene cinco universidades que cuentan con la escuela de Ingeniería Industrial: UPN, UCV, UNT, UPAO y UCT.

Solo en las dos primeras las escuelas de Ingeniería Industrial tienen el mayor número de estudiantes y más de 10 años de funcionamiento, esta razón limitó la investigación de campo, considerando también las facilidades de acceso.

Para el estudio se incluyeron Los estudiantes de Ingeniería Industrial del V, VI, VII y VIII ciclo de matrícula regular en el semestre académico 2017-I, de ambos sexos. Se excluyeron a los estudiantes no seleccionados para la muestra. Se excluyeron a los estudiantes no presentes, los que no terminaron el inventario y aquellos con más de una respuesta negativa al aplicar el inventario SRLI [16]. Las unidades muestrales se seleccionaron en forma aleatoria, según registros de estudiantes.

La población objeto de estudio fueron 280 estudiantes de la universidad $\mathrm{N}$ y 120 estudiantes de la universidad V. El tamaño de muestra se determinó para un nivel de confianza del $95 \%$, siendo 135 estudiantes, y de forma aleatoria se seleccionaron 94 estudiantes de la universidad $\mathrm{N}$ y 41 estudiantes de la universidad $\mathrm{V}$, según registros de estudiantes

\section{B. El modelo cíclico de fases de Zimmerman del aprendizaje autorregulado}

El Modelo cíclico de fases de Zimmerman, fue presentado por primera vez en el año 2000, [7].

Fase de planificación (Forethought phase).

En la fase inicial el estudiante se enfrenta por primera vez a la tarea, aquí analiza, evalúa su capacidad para realizarla con éxito, establece metas y planifica. El estudiante establece sus objetivos a partir de dos variables: los criterios de evaluación y el nivel de perfección que quiere alcanzar. Cuando sus expectativas de autoeficacia son altas, empleará las estrategias necesarias para enfrentar las dificultades que se presenten.

Así los estudiantes con metas de aprendizaje seleccionan y utilizan estrategias con aprendizajes más profundos, tienen actividades de reflexión más avanzados, aceptan los fracasos académicos, se recuperan y muestran mayor interés intrínseco por las tareas.

\section{Fase de ejecución (Performance phase).}

Durante la ejecución el estudiante mantiene la concentración y utiliza estrategias de aprendizaje adecuadas por dos motivos. Primero para no disminuir su interés y motivación y segundo, para alcanzar los objetivos de aprendizaje. Los principales procesos durante la ejecución son la auto observación y el auto control.

La monitorización metacognitiva o auto supervisión, consiste en que el estudiante compare lo que se está realizando con algún criterio que permita evaluar su ejecución. Para mantener el interés y la atención es necesario el control del entorno de trabajo, se alcanza cuando el estudiante crea un ambiente de trabajo con pocas distracciones, favoreciendo el desarrollo de la tarea y aumentando su efectividad.

Fase de autorreflexión (Self-reflection phase).

Durante esta fase el estudiante valora su tarea y se explica las razones de los resultados obtenidos, de esta manera justifica las causas de su éxito o fracaso y según su estilo atribucional, experimenta emociones positivas o negativas que logran influir en su motivación y en su capacidad de autorregulación en el futuro.

La autoevaluación es la valoración que un estudiante hace de su tarea como correcto o incorrecto en base a los criterios de calidad, que pueden fijarse al inicio de la actividad con la ayuda del docente de tal manera que el estudiante sepa cómo autoevaluar su tarea y de esta manera corregir los errores. En la medida en que los estudiantes sepan valorar sus éxitos y fracasos como ocasiones para mejorar de cómo reaccionar, pueden controlar las atribuciones que realizan para que estas sean adaptativas.

\section{Marco conceptual}

Se define ellos conceptos de aprendizaje autorregulado, metacognición, motivación y rendimiento académico.

\section{Aprendizaje autorregulado (SRL).}

Forma de aprendizaje que los estudiantes realizan, según el tipo de su motivación para aprender, utilizando una o más medidas autorreguladoras (de naturaleza cognitiva, metacognitiva, volitiva o conductual) y el seguimiento del progreso de su aprendizaje, [16].

\section{Metacognición.}

Es la capacidad de un estudiante para entender y regular sus pensamientos y acciones realizadas. Organiza planes de acción y selecciona estrategias para resolver problemas según sus necesidades, llevando a cabo o suspendiendo su aplicación cuando sea necesario, evalúa su eficacia y eficiencia, y determina si han alcanzado los objetivos.

\section{Motivación.}

Es un conjunto de procesos desarrollados por el estudiante en la activación, dirección y sostenimiento de la conducta.

\section{Rendimiento académico.}

Es la información cuantitativa y cualitativa del aprendizaje de un estudiante (habilidades, conocimientos, valores y aptitudes), a partir de la aplicación de un evaluativo.

D. Técnicas de procesamiento y análisis

Para el análisis de datos se empleará la estadística descriptiva y la inferencial. 


\section{Estadística descriptiva}

Se analiza los datos obtenidos con el Inventario de Aprendizaje Autorregulado, describiendo las puntuaciones, frecuencias, medidas de tendencia central, de variabilidad para cada variable; también análisis de fiabilidad de los datos (Alfa de Cronbach) y pruebas de paramétricas (Kolmogorov Smirnov por tener serie de datos mayor a 50).

\section{Estadística inferencial}

La prueba de hipótesis se realizó con la correlación lineal de Pearson entre las variables de estudio Aprendizaje Autorregulado y rendimiento Académico, luego que las pruebas paramétricas (Kolmogorov - Smirnov) confirmaron la normalidad en ambas variables.

\section{E. Técnicas e instrumentos de recojo de datos}

El instrumento que se utilizó es el Inventario del aprendizaje autorregulado [16]. La referencia [26], realizaron la validación del SRLI, el Inventario versión 3.0. El coeficiente de Alpha de Cronbach calculado fue de (.93) en total, que se califica de alta confiabilidad y consistencia.

La referencia [27] en su investigación realizada para su Maestría en Calidad de la Educación, realizó la traducción al español del Inventario de Autorregulación para el Aprendizaje (SRLI) [16], de esta versión se tomó como base para la medición en la presente investigación. La referencia [15] tomó la decisión de reducir el inventario de 80 a 60 ítems, reduciendo 05 ítems en forma proporcional de las 04 subescalas, y sobre todo los ítems que eran algo confusos. La puntuación máxima ya no fue de 400 puntos sino de 300 . El tiempo requerido para el desarrollo del inventario es de 15 a 20 minutos.

\section{Inventario de Aprendizaje Autorregulado (SRLI).}

El Inventario del aprendizaje autorregulado (SRLI) [16] se basó en el modelo (SESRL) [28]. Es el grado que desarrollan los estudiantes en sus propios procesos de aprendizaje.

Ejecutivo: Proceso de Ejecución, proceso metacognitivo. Cognitivo: Proceso cognitivo, almacenamiento y recuperación datos, tareas. Motivacional: Orientación hacia la meta, recompensa, capacidad. Control del ambiente: Empleo del medio ambiente, ayuda, tiempo y tarea.

Indicador: Puntaje obtenido por los estudiantes considerando la aplicación del Inventario de Aprendizaje Autorregulado (SRLI) [15]. Los ítems del inventario de aprendizaje autorregulado por subescalas fueron 15 items para cada subescala: ejecutiva, cognitiva, motivación y control del ambiente.

Los resultados en los ítems negativos, elaborados para evitar el sesgo en las respuestas del estudiante, según las subescalas, fueron cero items para ejecutiva, tres items para cognitiva, un item para motivación y cuatro items para control del ambiente [15].

\section{Confiabilidad del instrumento de estudio.}

Realizada la aplicación y depuración de las encuestas, se calculará los índices de confiabilidad. La confiabilidad se establece determinando el coeficiente de Alfa de Cronbach, que varía de 0 a 1, el valor 1 indica la mayor consistencia [29].

\section{Niveles de Rendimiento Académico}

Calificación final obtenido por los estudiantes en la escala vigesimal con dos decimales, referidos a las asignaturas y a su promedio respectivo. Indicador: Puntaje obtenido por los estudiantes considerando el promedio de las calificaciones obtenidas en los cursos de las diferentes asignaturas. Para la valoración del aprendizaje se tomó los criterios de [30].

\section{Escala Diagnóstica específica y general para el inventario del Aprendizaje Autorregulado. \\ El inventario del Aprendizaje Autorregulado permite} evaluar la forma de aprendizaje que los estudiantes realizan, Un cuadernillo formado por 60 enunciados; en donde 15 están relacionados a la medición de 4 subescalas. Adaptado [15]. El rango de valoración de cada enunciado es del 1 al 5, la mínima calificación es 60 puntos y la máxima 300 puntos.

\section{Escala de Correlación de Pearson R.}

Para realizar la prueba de las hipótesis se ha tomado la correlación lineal de Pearson $\mathrm{R}(-1 \leq \mathrm{R} \leq 1)$. Según la correlación lineal de Pearson nos indica que: si $\mathrm{R}=1$, entonces se dice que hay una correlación perfecta positiva el cual indica una variación proporcional, es decir, si la variable x aumenta, y aumenta de modo proporcional (esto es válido si x disminuye, $\mathrm{y}$ entonces disminuye). $\mathrm{Si} \mathrm{R}=-1$, una correlación perfecta negativa, esto es, si "x" aumenta, " $y$ " disminuye (válido, si " $x$ " disminuye, "y" aumenta) [31].

\section{RESULTADOS}

Se presentan y analizan los resultados, se determina la confiabilidad del instrumento para el estudio de la muestra, se somete a la prueba de Normalidad los datos recolectados, se determina los descriptivos estadísticos de las variables y se analiza según la prueba de hipótesis la correlación entre las variables.

\section{Presentación y análisis de resultados}

La muestra de estudio está formada por 135 estudiantes, el porcentaje de estudiantes por ciclo, el $16 \%$ fueron estudiantes del V ciclo, $14 \%$ de estudiantes del VI ciclo, el $45 \%$ por estudiantes del VII ciclo y $24 \%$ de los estudiantes del VIII ciclo.

La muestra de estudio fueron estudiantes entre 18 y 30 años, el porcentaje más resaltantes por edad fueron, el $24 \%$ de estudiantes con 20 años y $28 \%$ de los estudiantes con 21 años. La muestra de estudio estuvo formada por estudiantes de ambos sexos, el $51 \%$ fueron mujeres y el $49 \%$ hombres.

\section{Confiabilidad del instrumento de estudio}

La confiabilidad se estableció determinando el coeficiente de Alfa de Cronbach, que varía de 0 a 1 , el valor 1 indica la 
mayor consistencia. En general, según el alfa de Cronbach de la escala total y de cada una de las subescalas, evidencian que el SRLI es un instrumento idóneo para su aplicación en la muestra en estudio. Los resultados obtenidos para la escala que corresponde a la subescala Ejecutiva presenta una consistencia de nivel aceptable $(\alpha=0.726)$, lo mismo para la subescala Motivación presenta una consistencia de nivel aceptable $(\alpha=$ 0.720). La subescala Cognitiva presenta la consistencia es pobre $(\alpha=0.572)$, y la de Control del ambiente es cuestionable $(\alpha=0.6688)$.

\section{Prueba de hipótesis de Normalidad}

Es necesario explorar las variables cuantitativas para comprobar que se cumplen los requisitos que van a permitir aplicar las pruebas paramétricas. Para ello recurrimos a la prueba de Normalidad.

Cómo el número de observaciones superan el número de 50, se toma como referencia el método de Kolmogorov Smirnov.

TABLA I

PRUEBA DE NORMALIDAD ENTRE EL RENDIMIENTO ACADÉMICO Y PUNTAJE DEL INVENTARIO DEL APRENDIZAJE AUTORREGULADO Y LAS SUBESCALAS

\begin{tabular}{|l|l|l|l|}
\hline Variables & Estadístico & $\mathrm{gl}$ & Sig. \\
\hline Rendimiento & 0.077 & 135 & 0.048 \\
\hline Ejecutiva & 0.064 & 135 & 0.200 \\
\hline Cognitiva & 0.081 & 135 & 0.032 \\
\hline Motivación & 0.072 & 135 & 0.081 \\
\hline Control de ambiente & 0.061 & 135 & 0.200 \\
\hline Total & 0.082 & 135 & 0.027 \\
\hline
\end{tabular}

Fuente: Tomada de IBM SPSS Statistics 21

De acuerdo con los datos de la Tabla I, en todos los casos la Hipótesis H0 se acepta (Sig. > 0.01), porque existe alta probabilidad de seguir una distribución normal. Por tanto, los datos de las variables siguen una distribución Normal.

Descriptivos estadísticos de las variables.

Para determinar el nivel de aprendizaje autorregulado que tienen los estudiantes de Ingeniería Industrial de la muestra de estudio, se procedió a calcular la media aritmética, para cada una de las subescalas y la escala total, así como también los estadísticos del rendimiento académico.

TABLA II

PUNTAJE PROMEDIO DEL RENDIMIENTO ACADÉMICO Y DEL INVENTARIO DEL APRENDIZAJE AUTORREGULADO Y LAS SUBESCALAS.

\begin{tabular}{|l|l|l|l|}
\hline & Media & Desv.Tip. & Error tip. \\
\hline Rendimiento & 14.8 & 0.8 & 0.0691 \\
\hline Ejecutiva & 54.0 & 6.5 & 0.5597 \\
\hline Cognitiva & 54.6 & 5.2 & 0.4483 \\
\hline Motivación & 53.7 & 6.6 & 0.5639 \\
\hline Control de Ambiente & 55.0 & 6.0 & 0.5167 \\
\hline Total & 217.3 & 21.4 & 1.8397 \\
\hline
\end{tabular}

Fuente: Tomada de IBM SPSS Statistics 21

Los resultados mostrados en la Tabla II para el Rendimiento Académico alcanzado por los estudiantes de
Ingeniería Industrial (14.8), se ubican en el nivel medio (13.00 a 14.99) [30].

En cuanto al nivel del Aprendizaje Autorregulado alcanzado los resultados mostrados en la Tabla II se ubican en el nivel medio para las subescalas (45.0 a 59.9), así para la escala total (217.3) se ubica en el nivel medio (181 a 240) [15].

\section{Prueba de hipótesis de Correlación entre variables}

A continuación, se realiza el análisis del coeficiente de correlación de Pearson $\mathrm{R}$ para las hipótesis formuladas, siguiendo el siguiente esquema:

H0: $R=0$; Significa que ambas variables no están linealmente correlacionadas.

H1: R > 0; Significa que ambas variables están linealmente correlacionadas.

Si el valor de 'P' o Sig.(bilateral) $>0.01$ La Hipótesis H0 se acepta.

Si el valor de 'P' o Sig.(bilateral) < 0.01 La Hipótesis H0 se rechaza.

TABLA III

CORRELACIÓN ENTRE EL RENDIMIENTO ACADÉMICO Y EL APRENDIZAJE AUTORREGULADO Y LAS SUBESCALAS EN LOS ESTUDIANTES DE INGENIERÍA INDUSTRIAL

\begin{tabular}{|l|l|l|l|l|}
\hline Subescala & Estadístico & Univ. V & Univ. N & Global \\
\hline & Estudiantes & 41 & 94 & 135 \\
\hline \multirow{3}{*}{ Total } & Pearson & 0.777 & 0.854 & 0.822 \\
\cline { 2 - 5 } & Sig.(bilat) & 0.000 & 0.000 & 0.000 \\
\hline \multirow{2}{*}{ Ejecutiva } & Pearson & 0.709 & 0.758 & 0.736 \\
\cline { 2 - 5 } & Sig.(bilat) & 0.000 & 0.000 & 0.000 \\
\hline \multirow{2}{*}{ Motivación } & Pearson & 0.658 & 0.742 & 0.707 \\
\cline { 2 - 5 } & Sig.(bilat) & 0.000 & 0.000 & 0.000 \\
\cline { 2 - 5 } & Pearson & 0.653 & 0.758 & 0.716 \\
\cline { 2 - 5 } $\begin{array}{l}\text { Control de } \\
\text { ambiente }\end{array}$ & Sig.(bilat) & 0.000 & 0.000 & 0.000 \\
\cline { 2 - 5 } & Pearson & 0.775 & 0.715 & 0.733 \\
\hline \multirow{2}{*}{$*$} & Sig.(bilat) & 0.000 & 0.000 & 0.000 \\
\hline
\end{tabular}

Fuente: Tomada de IBM SPSS Statistics 21

En la Tabla III, el valor de 'P' o Sig.(bilateral) es menor que el nivel crítico 0.01 , tanto para los datos de estudiantes de las universidades $\mathrm{V}, \mathrm{N}$ y en conjunto. $(0.000<0.01)$. Por tanto, la Hipótesis $\mathrm{H} 0$ se rechaza, porque existe baja probabilidad de que $\mathrm{R}$ sea igual a 0, Esto quiere decir que las variables de aprendizaje autorregulado y el rendimiento académico están significativamente correlacionadas. También se aprecia que la correlación lineal de Pearson para los datos de estudiantes de las universidades $\mathrm{V}, \mathrm{N}$ y Global, se consideran que tienen una correlación positiva media [31]. 


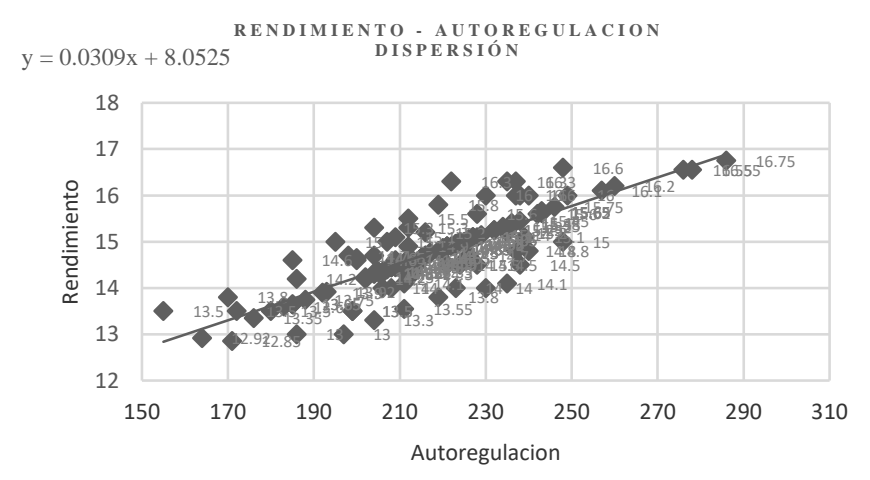

Fig.1 Diagrama de dispersión entre Rendimiento y Autorregulación

En el diagrama de dispersión de la Figura 1, se observa la relación entre el rendimiento académico y la autorregulación.

\section{DISCUSIÓN DE RESULTADOS}

Se presenta la discusión de los resultados para la correlación entre variables, incluyendo las subescalas para el aprendizaje autorregulado, y de los promedios de las variables alcanzados por los estudiantes de la muestra analizada.

Correlación entre Aprendizaje autorregulado total y Rendimiento académico.

Los resultados para la hipótesis general demuestran una correlación positiva considerable entre el aprendizaje autorregulado y el rendimiento académico en los estudiantes de Ingeniería Industrial [31]. En la Tabla III, se aprecia que la correlación lineal de Pearson es $\mathrm{R}=0,777$ a un nivel de significancia de 0.01, para la universidad V. Para la universidad $\mathrm{N}$ en la Tabla III, se tiene que $\mathrm{R}=0,854$ a un nivel de significancia de 0,01 y en conjunto $\mathrm{R}=0.822$. En la referencia [15] la correlación lineal de Pearson es $\mathrm{R}=0,596$ y en la investigación de Norabuena (2011) la correlación lineal de Pearson es $R=0,6769$. En ambos casos una correlación positiva media, se evidencia una diferencia que se explica por el cuidado en la selección de la muestra aleatoria, y la orientación debida, por tanto, una mejor comprensión del estudio por parte de los estudiantes.

Correlación entre las subescalas del Aprendizaje autorregulado y Rendimiento académico.

Como se muestra en la Tabla III, los resultados en la matriz de correlaciones del Inventario del aprendizaje autorregulado, para todas las subescalas (Ejecutiva, Cognitiva, Motivación y Control de ambiente) y el rendimiento académico en la universidad V varía entre 0,653 y 0,775 , con un nivel de significancia de 0,01 . Mientras que en la universidad $\mathrm{N}$ se tiene que la correlación varía entre 0,715 y 0,758 , en su conjunto $\mathrm{R}$ varía entre 0,716 y 0.736 , existe una correlación positiva media [31]. En la referencia [17], la correlación lineal de Pearson es $\mathrm{R}=0,572$ y en la referencia [15] la correlación lineal de Pearson varía entre 0,498 y 0,572. En ambos casos una correlación positiva media, se evidencia qué si el nivel de subescalas Ejecutiva, Cognitiva, Motivación y Control de ambiente del Aprendizaje autorregulado aumenta, el Rendimiento académico se eleva.

\section{Promedios Aprendizaje autorregulado}

Con relación al aprendizaje autorregulado de los estudiantes de Ingeniería Industrial, se encontró que el promedio (217.28) presenta un nivel medio en esta variable, tal como se muestra en la Tabla II [15]. Se considera un desarrollo adecuado en las subescalas Ejecutiva, Cognitiva, de Motivación, y de Control del ambiente, aunque sin alcanzar un nivel satisfactorio. Los resultados obtenidos son similares al estudio sobre aprendizaje autorregulado y rendimiento académico en estudiantes de Ingeniería Industrial de la Universidad Tecnológica del Perú [15], donde reportó que el promedio de los estudiantes se sitúa en el nivel medio del aprendizaje autorregulado.

\section{Promedios Rendimiento académico}

En cuanto a los resultados alcanzados en el rendimiento académico, el promedio de los estudiantes (14.8) según [30], alcanza el nivel medio en esta variable, tal como se muestra en la Tabla II. Lo que significa, que el logro académico en las asignaturas que siguen los estudiantes de la muestra no alcanza niveles altos. Estos resultados son similares al estudio [15]; donde reportó que el promedio de los estudiantes se sitúa en el nivel medio en rendimiento académico.

\section{CONCLUSIONES}

Considerando la correlación de Pearson para el aprendizaje autorregulado medido con SRLI y el rendimiento académico en estudiantes de Ingeniería Industrial en universidades en Trujillo 2017, se concluye que existe una relación positiva media (R: 0.822). Esto es importante para las estrategias de aprendizaje que el docente y el estudiante deben implementar, ya que la autorregulación es el control de la acción que integra: el conocimiento metacognitivo, la regulación de la cognición y motivación, [6].

Considerando la correlación de Pearson para todas las subescalas del aprendizaje autorregulado, (Ejecutiva, Cognitiva, Motivación y Control de ambiente) medido con SRLI [15] y el rendimiento académico, se concluye que existe una relación positiva media (R: $0.716-0.736)$. Estos resultados implican que, durante la ejecución el estudiante mantiene la concentración y utiliza estrategias de aprendizaje adecuadas por dos motivos; primero para no disminuir su interés y motivación y segundo, para alcanzar los objetivos de aprendizaje [7].

Considerando el promedio total para el aprendizaje autorregulado medido con SRLI y de las subescalas (53.7 55.0) se concluye que los estudiantes tienen un nivel medio (217.3). La importancia de los resultados es por las características que diferencian a los estudiantes que autorregulan su aprendizaje de aquellos que no lo hacen, debido a que emplean estrategias cognitivas que les ayuda a atender, 
transformar, organizar, elaborar y recuperar la información, [32].

Considerando el promedio del rendimiento académico en la población estudiada, se concluye que existe un nivel medio (14.8). Los resultados de la investigación implican un apoyo directo a los docentes en su búsqueda de trasformar su rol en el aula, de las relaciones entre docente y estudiantes o entre los propios estudiantes. Un conocimiento sobre el proceso de facilitación, por medio de estrategias de aprendizaje que el docente y el estudiante deben implementar. Estos resultados evidenciaron durante la pandemia del COVID-19, qué para mejorar el nivel del aprendizaje autorregulado, las universidades de Trujillo deben contemplar en su mejora continua, estrategias innovadoras en las escuelas de Ingeniería Industrial, en consecuencia, también se obtendrá mejoras en el rendimiento académico.

\section{REFERENCIAS}

[1] Hong, J. C., Lee, Y. F., \& Ye, J. H. (2021). Procrastination predicts online self-regulated learning and online learning ineffectiveness during the coronavirus lockdown. Personality and individual differences, 174, 110673. https://doi.org/10.1016/j.paid.2021.110673

[2] Jansen, R. S., van Leeuwen, A., Janssen, J., Conijn, R., \& Kester, L. (2020). Supporting learners' self-regulated learning in Massive Open Online Courses. Computers \& Education, 146, 103771. https://doi.org/10.1016/j.compedu.2019.103771

[3] Castejón Costa J. L. (2015). Aprendizaje y rendimiento académico. Alicante. España: Editorial Club Universitario.

[4] Rodríguez, S., Fita, E., y Torrado, M. (2004). El rendimiento académico en la transición secundaria-universidad. Revista de educación, 334, 391-414.

[5] Santander, O. A. E. (2011). El rendimiento académico, un fenómeno de múltiples relaciones y complejidades. Revista Vanguardia Psicológica clínica teórica y práctica, 2(2), 144-173.

[6] Lanz, M. Z. (2006). El aprendizaje autorregulado (Vol. 63). Noveduc Libros. Buenos Aires, Argentina.

[7] Panadero, E., y Tapia, J. (2014). ¿Cómo autorregulan nuestros estudiantes? Revisión del modelo cíclico de Zimmerman sobre autorregulación del aprendizaje. Anales de Psicología, 30(2). Servicio de Publicaciones de la Universidad de Murcia. Murcia (España). Recuperado de http://revistas.um.es/analesps/article/view/analesps.30.2.167221/161091

[8] Zimmerman, B. J., \& Moylan, A. R. (2009). Self-regulation: Where metacognition and motivation intersect. In D. J. Hacker, J. Dunlosky \& A. C. Graesser (Eds.), Handbook of Metacognition in Education (pp. 299315). New York: Routledge.

[9] Contrera Y. y Rodríguez A. (2012, octubre). Aprendizaje auto-regulado como competencia para el aprovechamiento de los estilos de aprendizaje en estudiantes de educación superior. Revista Estilos de Aprendizaje, V.5, n.10, n.10. Recuperado de http://catedra.ruv.itesm.mx/bitstream/987654321/707/1/Aprendizaje\%20a uto-

regulado $\% 20$ como $\% 20$ competencia\%20para\%20el\%20aprovechamiento $\% 20 \mathrm{de} \% 20 \mathrm{los} \% 20$ estilos $\% 20 \mathrm{de} \% 20$ aprendizaje $\% 20$ en $\% 20$ estudiantes $\% 2$ 0de\%20educacion\%20superior.pdf

[10]Chocarro, E., González-Torres, M. C., y Sobrino-Morrás, Á. (2007). Nuevas orientaciones en la formación del profesorado para una enseñanza centrada en la promoción del aprendizaje autorregulado de los estudiantes. ESE. Estudios sobre educación, 2007, $N^{o}$ 12, pp. 8I-98. Recuperado de http://dadun.unav.edu/handle/10171/9003

[11]Quintana-Terés, María C. (2014). El aprendizaje autorregulado en estudiantes de educación superior. (Tesis de Doctorado). Universidad Iberoamericana Puebla, México.

[12]Zimmerman, B. J., Kitsantas, A., y Campillo, M. (2005). Evaluación de la autoeficacia regulatoria: una perspectiva social cognitiva. Revista Evaluar, 5 .
[13]Monterroso Martini Susy Candelaria (2015) Aprendizaje Autorregulado y su incidencia en el Rendimiento Académico de los adolescentes. (Tesis de Maestría). Universidad Rafael Landívar. Guatemala.

[14]Torre Puente, J. C. (2007). Una triple alianza para un aprendizaje universitario de calidad. Madrid: Edit. Comillas, Madrid.

[15]Norabuena Penadillo, R. M. (2011). Relación entre el aprendizaje autorregulado y rendimiento académico en estudiantes de enfermería y obstetricia de la Universidad Nacional Santiago Antúnez de MayoloHuaraz. (Tesis de Maestría). Universidad Nacional Mayor De San Marcos. Perú.

[16]Lindner, R. W., \& Harris, B. (1992). Self-Regulated Learning and Academic Achievement in College Students.

[17]Valqui Zuta, E. (2008). Aprendizaje autorregulado y rendimiento académico en estudiantes de la especialidad de Ingeniería Industrial de la Universidad Tecnológica del Perú. (Tesis de Maestría). Universidad Nacional Mayor De San Marcos. Perú.

[18]Arias W., Zegarra J. y Velarde O. (2014, octubre 10). Estilos de aprendizaje y meta cognición en estudiantes de psicología de Arequipa. La Scientific Electronic Library Online - Scielo Perú, liberabit. vol.20 n.2 Lima jul. /dic. 2014, $\quad n .2$ Recuperado de http://www.scielo.org.pe/scielo.php?pid=S172948272014000200008\&script=sci_arttext\&tlng=en

[19]Rebaza Villacorta, E. B. (2017). Relación entre estrategias metacognitivas, aprendizaje autorregulado y autoestima en los estudiantes en el instituto superior pedagógico Indoamérica 2011. (Tesis de Maestría). Universidad Privada Antenor Orrego. Perú.

[20]O'Neil Jr, H. F., \& Abedi, J. (1996). Reliability and validity of a state metacognitive inventory: Potential for alternative assessment. The Journal of Educational Research, 89(4), 234-245.

[21]Coopersmith, S. (1967). Coopersmith self-esteem inventory form A. SelfEsteem Institute.

[22]Domínguez Vásquez, R. P. (2015). Estrategias didácticas y rendimiento académico de los estudiantes de educación secundaria de las instituciones educativas del distrito de Taurija - La Libertad - 2013. (Tesis de Maestría). Universidad Nacional de Educación Enrique Guzmán y Valle.

[23]Diseño Curricular Nacional (2009) Resolución Ministerial No 0440-2008ED. https://cdn.www.gob.pe/uploads/document/file/148150/_0440-2008ED_-_15-10-2012_09_17_49_-RM-0440-2008-ED.pdf

[24]SUNEDU. (2017). Informe bienal sobre la realidad universitaria peruana. 05/02/2018, de Superintendencia Nacional de Educación Superior Universitaria Sitio web: https://www.sunedu.gob.pe/informe-bienal-sobrerealidad-universitaria/

[25]SUNEDU. (2018). Estudiantes matriculados de pregrado por programa de estudio. 05/02/2018, de Superintendencia Nacional de Educación Superior Universitaria Sitio web: https://www.sunedu.gob.pe/sibe/

[26]Reinhard, L. W., Bruce, R. H., \& Wayne, I. G. (1996). The Design and Development of The Self-Regulatred Learning Inventory: A status report. In Documento presentado en Annual Meeting of the American Educational Research (Vol. 77, pp. 8-12).

[27]Espinosa, A. L. R. (2002). Diferencias en las estrategias y atribuciones de aprendizaje autorregulado de estudiantes de nuevo ingreso a nivel Licenciatura de la UDLA-P. (Tesis de Maestría). Universidad de las Américas Puebla. México.

[28]Zimmerman, B. J., \& Pons, M. M. (1986). Development of a structured interview for assessing student use of self-regulated learning strategies. American educational research journal, 23(4), 614-628.

[29]George, D. y Mallery, P. (2003). SPSS for Windows step by step: A Simple Guide and Reference. 11.0 Update (4. ${ }^{\mathrm{a}}$ ed.). Boston: Allyn \& Bacon.

[30]Reyes Murillo, E. (1998). Influencia del programa curricular y del trabajo docente en el aprovechamiento escolar en Historia del Perú de los estudiantes de $3^{\circ}$ Grado de Ed. Secundaria (Tesis doctoral). Lima: Universidad Nacional Mayor de San Marcos).

[31]Sampieri, R. H., Collado, C. F., y Lucio, M. D. (2014). Metodología de la investigación (sexta edición ed.). México DF: Mc-Graw Hill.

[32]Montalvo, F. T., y Torres, M. C. G. (2004). El aprendizaje autorregulado: presente y futuro de la investigación. Revista electrónica de investigación psicoeducativa, 2(1), 1-34. Universidad de Navarra. Recuperado de http://portalliceo.com/Administrador/documentos/APRENDIZAJE\%20A UTORREGULADO.pdf 